\title{
Treatment of vocal fatigue in teachers
}

\begin{abstract}
Teaching is a vocally demanding profession that demands long periods of speaking. Teachers are often exposed to some factors that are detrimental to their vocal health so as a result they report symptoms of vocal fatigue. This study determined the effectiveness as well as the efficacy of Vocal Hygiene Training (VHT) and Resonant Voice Therapy (RVT) in reducing self reported symptoms of vocal fatigue among teachers. The study also examined the moderating effect of gender and years of teaching experience on the treatments. Thirty teachers with vocal fatigue were purposively selected from four secondary schools in Oyo state and were exposed to three treatment conditions (VHT, RVT and Control group) which lasted for seven weeks. Two research instruments were used: Vocal Screening Structured Interview and Vocal Fatigue Index $(r=0.76)$. Three hypotheses were tested at 0.05 level of significance. Data were analysed using Analysis of Covariance, and Bon feroni was used as post hoc. The participants trained with Resonant Voice Therapy experienced significant reduction in self perceptive symptoms of vocal fatigue with adjusted mean score (24.01) followed by Vocal Hygiene Training (36.68). Therefore, Resonant Voice Therapy and Vocal Hygiene Training should be adopted by professional speech and language pathologists in the treatment of vocal fatigue among teachers.
\end{abstract}

Keywords: vocal hygiene training, resonant voice therapy, gender, years of teaching experience, vocal fatigue, teachers
Volume 7 Issue I - 2017

Gerald Chukwudi Imaezue, Moji Oyebola

Department of Special Education University of Ibadan, Nigeria

Correspondence: Gerald Chukwudi Imaezue Speech

Pathology and Audiology Unit Department of Special Education University of Ibadan Nigeria West Africa, Tel +234703342398I, Email geraldimaezue@gmail.com

Received: June 16,2016 | Published: April 26, 2017
Abbreviations: RVT, resonant voice therapy; VHT, vocal hygiene training; VFI, voice fatigue index; VSSI, vocal screening structured interview;

\section{Introduction}

Teachers are one of the largest professional groups with voice as their main work tool. Teaching is a vocally demanding profession that demands long periods of speaking plus the teacher's voice competing with environmental noise for students' attention, inadequate ventilation as a result of class overcrowding, few opportunities for resting voice and extra vocal burdens such as tutoring, parent-teacher meetings and personal vocal demands for example socializing in noisy environments during weekends.

A common complaint by teachers and other occupational voice users is vocal fatigue. ${ }^{1}$ Hunter $^{2}$ defined vocal fatigue as a condition resulting from an overused or under-recovered voice caused by excessive tissue vibration or repeated vocal fold posturing. However, there has not been a universally accepted definition for vocal fatigue. In their review of vocal fatigue, Vocal fatigue is often used by patients as a lay description of the symptoms of vocal loading, and it is also used by medical practitioners and voice professionals as a clinical diagnosis of the result of these symptoms. Vocal fatigue is common among teachers especially because of the nature of their job and their work environment.

In Nigeria, teachers use their voice for hours in acoustically poor designed learning environments and are highly susceptible to vocal fatigue which can be due to vocal abuse. Teachers are often subject to heavy voice use or voice overload, exposure to irritants, the need to raise vocal volume and limited vocal stress recovery time. Vocal dysfunction may not only have a great negative impact on the teachers' well-being at work but also on the classroom communication. Hence, it may also disturb the students' learning process.

The anatomy and physiology of the human speech-voice mechanism differ on the ground of gender. Studies have shown that the vocal cords of females vibrate faster than that of male. The anatomy of the larynx of females shows that it is thinner and narrower than that of males because the larynxes of males undergo significant anatomical and physiological changes during puberty when compared to their female counterparts. For the purpose of this study, it is assumed that gender might contribute to the incidence as well as severity of symptoms of vocal fatigue among teachers. Vocal loading has been identified as one of the major factors responsible for vocal fatigue among teachers. Vocal loading refers to stress inflected on the speech organs as a result of speaking for a long period of time. By implication, vocal loading is reflected in the years of teaching experience which may contribute to vocal fatigue as well as the severity of the symptoms of vocal fatigue among teachers.

With no unambiguous definition or etiology of vocal fatigue, it has been difficult to identify which indices that could be used to consistently and accurately measure the occurrence, symptoms and severity of vocal fatigue. Measures often used to evaluate vocal fatigue are acoustic analysis, aerodynamic analysis, laryngeal appearance and self-reports. ${ }^{3}$ An important instrument designed to measure self reported symptoms of vocal fatigue is the Vocal Fatigue Index. Voice Fatigue Index (VFI) is an instrument constructed by Nanjudeswaran et al., ${ }^{3}$ This is a clinical measure that can estimate perceived vocal fatigue and it can be reliably used to compare across patients with vocal fatigue. ${ }^{3}$ This index encompasses the symptoms typically associated with and self-reported by individuals with vocal fatigue.

Preventive and intervention strategies are recommended to reduce the risk of voice fatigue which might be as a result of the aforementioned factors among teachers. One of such suggested methods of primary prevention cum intervention is vocal hygiene training. Vocal hygiene training involves training professional voice users on healthy habits and behaviours that promote vocal health. Various studies have shown positive acoustic and perceptual effects of voice hygiene training and voice therapy. ${ }^{4}$ Voice hygiene education as such has also been reported to have positive impact on the treatment of voice problems among professional voice users. ${ }^{5}$ 
In a six-week course for professional voice users consisting of six weekly sessions of two hours each, the overall aim was to increase the participants' ability to use their vocal apparatus and to make them more aware of the context in which they use their voices. ${ }^{6}$ The participants' subjective opinion was that they benefited greatly from the course. A follow-up study reporting the results from the acoustic analyses of the recordings made of the participants' voices before and after the course supported this opinion.?

Most studies focused only on the use of indirect treatments, for example vocal hygiene training, to the neglect of direct treatments as the sole remediation of self reported symptoms of vocal fatigue among teachers. The researcher, through this study, explored the effectiveness and efficacy of both indirect treatment (vocal hygiene training) and direct treatment (resonant voice therapy) in reducing self perceived symptoms of vocal fatigue among teachers. This study also provided relevant information on the effects of gender and years of teaching experience on treatment of self perceptive symptoms of vocal fatigue among teachers.

\section{Purpose of the study}

The objective of the study was to determine the efficacy of treatments (vocal hygiene training and resonant voice therapy) on self perceptive symptoms of vocal fatigue among teachers and to determine the interaction effect of the moderating variables on treatments of self perceptive symptoms of vocal fatigue among teachers;

\section{Hypotheses}

The following hypotheses were tested at 0.05 level of significance

a. There is no significant main effect of treatments (vocal hygiene training and resonant voice therapy) on self perceptive symptoms of vocal fatigue among teachers.

b. There is no significant interaction effect of treatments (VHT and RVT) and gender on self perceptive symptoms of vocal fatigue among teachers.

c. There is no significant interaction effect of treatments (VHT and RVT) and years of teaching experience on self perceptive symptoms of vocal fatigue among teachers. 4

\section{Methodology}

\section{Research design}

The study adopted a pretest-posttest, control group quasi experimental research design with a $3 \times 3 \times 1$ factorial matrix.

Participants: The participants of the study comprised all secondary school teachers (male and female) screened with symptoms of vocal fatigue in Ibadan, Oyo Stage, Nigeria. The study made use of purposive sampling technique to select the participants for the study.

Inclusion and exclusion criteria: These includes employment in a teaching occupation; over 5years teaching experience; incidence of vocal fatigue; normal intelligence as evidenced by graduation from an accredited college or university and English Language as second language. Participants who were deaf or hard of hearing or recipients of previous voice therapy were excluded from the study.

\section{Research instruments}

The researchers made use of two instruments; Vocal Screening Structured interview (VSSI) and Vocal Fatigue Index (VFI).
Vocal screening structured interview: A structured interview was designed by the researcher to elicit information about the commencement and history of the voice difficulty, relevant medical information, the domestic situation, participant's lifestyle and any trauma that may affect the voice.

Vocal fatigue index (VFI): The Voice Fatigue Index (VFI) which was adapted for this study is a standardised instrument constructed by Nanjudeswaran et al., ${ }^{3}$ The instrument includes 25 items to measure self reported symptoms of vocal fatigue. A 5 point scale was used to rate each statement as it reflects the participants' experience with their voice $(0=$ Never, $1=$ Almost Never, $2=$ Sometimes, $3=$ Almost Always, 4=Always).

Nanjudeswaran et al., ${ }^{3}$ report Cronbach's alpha internal consistency reliability of 0.91 for the total score of VFI. Test-retest reliability was measured in 10secondary school teachers with vocal fatigue across four weeks and the reliability was 0.76 for the total VFI score.

\section{Treatment package}

The treatments were carried out three (3) times a week for a period of seven weeks. Two weeks were used to train the research assistants; three weeks were used for the treatments, while two weeks were used for pretest and the posttest of self-perceptive symptoms of vocal fatigue among the participants. The Vocal Hygiene Training programme was developed by the researchers. The Resonant Voice Therapy protocol was adapted from Stemple et al., ${ }^{8}$ Clinical Voice Pathology guide on Resonant Voice Therapy.

Procedure for vocal hygiene training: Vocal hygiene training focuses on developing healthy vocal habits among the participants to help improve or maximise their vocal health. The treatment package was delivered through the expository method trained the participants via following modules; process of voice production, vocal abuse, effect of throat clearing and throat hydration, the need for adequate sleep and rest and effect of the use of low pitch and vocal fry. Instructional materials were used to supplement and aid treatment delivery. The researcher explained and modeled some focal hints of vocal hygiene to the participants and the learning objectives were evaluated.

Procedure for resonant voice therapy: RVT is designed to improve voice production in professional voice users with the least vocal effort. Its major goal is to establish and maintain forward voice behaviour or forward focus among the participants. The procedure includes contingent imitation in which the participants imitate the vocalization of the therapist. The research assistant employed the following techniques: describe action to be imitated, describe imitation, model an action or exercise the participants will imitate, use simulation for environment and emotional states, use wait time: Give the participants the opportunity to imitate the action spontaneously and give daily assignments.

\section{Data analysis}

Data obtained were analysed using Analysis of Covariance and Bon feroni post-hoc analysis.

\section{Results}

\section{Hypothesis I}

There is no significant main effect of treatments on self perceptive symptoms of vocal fatigue among teachers.

Table 1 revealed the main effect of treatment on self perceptive symptoms of vocal fatigue among teachers; $(\mathrm{F}(2,25)=40.402, \mathrm{p}<$ 
$0.05, \eta 2=0.764)$. Hence the null hypothesis was rejected. Therefore there was a significant main effect of treatment on self perceptive symptoms of vocal fatigue among teachers. This implies that there were variations in different treatment groups. Partial Eta-square revealed that the group difference accounted for $76.4 \%$ variance in their self perceptive symptoms of vocal fatigue. To further explain the differences in the groups self perceptive symptoms of vocal fatigue bonferoni pair wise comparison was carried out.

Table 2 revealed that after controlling for pretest vocal fatigue score. RVT group had the lowest self perceptive symptoms of vocal fatigue score (adjusted-mean= 24.01), followed by VHT group (adjusted-mean $=36.68$ ) and control group (adjusted-mean=62.04). This implies that resonant voice therapy was more effective in reducing self perceptive symptoms of vocal fatigue than other interventions. The overall coefficient of determination of the model reveals that the group difference accounted for $78.8 \%$ (Adjusted R Squared $=0.788$ ) variance in self perceptive symptoms of vocal fatigue among teachers (Table $3 \& 4$ )

Table I ANCOVA summary table reveals main effects of treatments on self perceptive symptoms of vocal fatigue among teachers

\begin{tabular}{lllllll}
\hline Source & Type III Sum of Squares & Df & Mean Square & F & Sig. c & Partial Eta Squared \\
\hline Corrected Model & 8774.756 & 3 & 2924.919 & 35.743 & 0 & $0.81 \mathrm{I}$ \\
Intercept & 1281.184 & $\mathrm{I}$ & 1281.184 & 15.656 & $0.00 \mathrm{I}$ & 0.385 \\
Pretest & 144.669 & $\mathrm{I}$ & 144.669 & 1.768 & 0.196 & 0.066 \\
Treatment & 6612.299 & 2 & 3306.149 & 40.402 & 0 & 0.764 \\
Error & 2045.796 & 25 & 81.832 & & & \\
Total & 60393 & 29 & & & & \\
Corrected Total & 10820.55 & 28 & & & & \\
\hline
\end{tabular}

R Squared $=.81 \mathrm{I}($ Adjusted R Squared $=.788)$

Table 2 Pair wise comparison showing differences on groups self perceptive symptoms of vocal fatigue

\begin{tabular}{lllllll}
\hline (I) Treatment & (J) Treatment & C & & & \multicolumn{2}{c}{ 95\% Confidence Interval for Differencec } \\
\hline & & Mean Difference (I-J) & Std. Error & Sig. c & Lower Bound & Upper Bound \\
\hline Control Group & VHT Group & 25.357 & 9.059 & 0.04 & 0.955 & 49.759 \\
& RVT Group & 38.024 & 4.78 & 0 & 25.15 & 50.899 \\
VHT Group & Control Group & -25.357 & 9.059 & 0.04 & -49.759 & -0.955 \\
& RVT Group & 12.667 & 8.516 & 0.473 & -10.273 & 35.607 \\
RVT Group & Control Group & -38.024 & 4.78 & 0 & -50.899 & -25.15 \\
& VHT Group & -12.667 & 8.516 & 0.473 & -35.607 & 10.273 \\
\hline
\end{tabular}

R Squared $=.81 \mathrm{I}$ (Adjusted R Squared $=.788$ )

Table 3 ANCOVA summary table showing interaction effects of treatment and gender on self perceptive symptoms of vocal fatigue among teachers

\begin{tabular}{|c|c|c|c|c|c|c|}
\hline Source & Type III Sum of Squares & Df & Mean Square & $\mathbf{F}$ & Sig. & Partial Eta Squared \\
\hline Corrected Model & 8925.647 & 6 & 1487.608 & $|7.27|$ & 0 & 0.825 \\
\hline Intercept & II34.048 & I & 1134.048 & 13.166 & 0.001 & 0.374 \\
\hline Pretest & 185.178 & I & 185.178 & 2.15 & 0.157 & 0.089 \\
\hline Treatment & 6343.216 & 2 & 3171.608 & 36.823 & 0 & 0.77 \\
\hline Gender & 35.639 & I & 35.639 & $0.4 \mid 4$ & 0.527 & 0.018 \\
\hline Treatment * Gender & 128.11 & 2 & 64.055 & 0.744 & 0.487 & 0.063 \\
\hline Error & 1894.905 & 22 & 86.132 & & & \\
\hline Total & 60393 & 29 & & & & \\
\hline Corrected Total & 10820.55 & 28 & & & & \\
\hline
\end{tabular}

R Squared $=.825$ (Adjusted R Squared $=.777$ )

Table 4 ANCOVA summary table showing interaction effects of treatment and years of teaching experience on self perceptive symptoms of vocal fatigue among teachers

\begin{tabular}{|c|c|c|c|c|c|c|}
\hline Source & Type III Sum of Squares & Df & Mean Square & $\mathbf{F}$ & Sig. & Partial Eta Squared \\
\hline Corrected Model & 9316.239 & 8 & 1164.53 & 15.483 & 0 & 0.861 \\
\hline Intercept & 684.641 & 1 & 684.641 & 9.102 & 0.007 & 0.313 \\
\hline Pretest & 145.428 & 1 & 145.428 & 1.933 & 0.18 & 0.088 \\
\hline Treatment & $4 \mid 45.423$ & 2 & 2072.711 & 27.557 & 0 & 0.734 \\
\hline Teaching Experience & 140.3 & 2 & 70.15 & 0.933 & $0.4 I$ & 0.085 \\
\hline Treatment and Teaching Exp & 269.217 & 3 & 89.739 & 1.193 & 0.338 & 0.152 \\
\hline Error & 1504.312 & 20 & 75.216 & & & \\
\hline Total & 60393 & 29 & & & & \\
\hline Corrected Total & 10820.55 & 28 & & & & \\
\hline
\end{tabular}

R Squared $=.86 \mathrm{I}($ Adjusted R Squared $=.805$ ) 


\section{Hypothesis 2}

There is no significant interactive effect of treatment and gender on self perceptive symptoms of vocal fatigue among teachers.

Table 5 revealed the interactive effect of treatment and gender on self perceptive symptoms of vocal fatigue among teachers; $(F(2,22)=$ $0.744, p>0.05, \eta 2=0.063)$. Hence the null hypothesis was not rejected. Therefore there is no significant interactive effect of treatment and gender on self perceptive symptoms of vocal fatigue among teachers. This implies that gender does not determine the effect of treatments on self perceptive symptoms of vocal fatigue among teachers. Partial Etasquare revealed that gender interaction with treatment accounted for $6.3 \%$ variance in teachers self perceptive symptoms of vocal fatigue.

\section{Hypothesis 3}

There is no significant interactive effect of treatment and years of teaching experience on self perceptive symptoms of vocal fatigue among teachers.

Table 6 revealed the interaction effect of treatment and teaching years of experience on self perceptive symptoms of vocal fatigue among teachers; $(\mathrm{F}(3,20)=1.193, \mathrm{p}>0.05, \eta 2=0.152)$ Hence the null hypothesis was not rejected. Therefore, there was no significant interaction effect of treatment and years of teaching experience on self perceptive symptoms of vocal fatigue among teachers. This implies that years of teaching experience does not significantly moderate the treatment of gender on self perceptive symptoms of vocal fatigue among teachers. Partial Eta-square revealed that years of teaching experience interaction with treatment accounted for $15.2 \%$ variance in teachers self perceptive symptoms of vocal fatigue.

\section{Discussion of findings}

The results of the findings revealed that there was significant effect of treatments (vocal hygiene training and resonant voice therapy) on self perceptive symptoms of vocal fatigue among teachers. Thus, hypothesis one was rejected. This finding is in agreement with Roy et al., ${ }^{9}$ who observed the efficacy of resonant voice therapy as treatment for voice disorders in teachers. It is also in line with the findings of Chan $^{5}$ who found that kindergarten teachers who underwent vocal hygiene training showed significant voice improvement.

The results also revealed that resonant voice therapy was the most effective treatment and this corroborates the findings of Stemple et al., ${ }^{10}$ which stated that the goal of RVT is to achieve the strongest and cleanest possible voice with the least effort and impact between the vocal folds to minimize the likelihood of injury and maximise the likelihood of vocal health. This is also in line with the findings of Hunter \& Titze ${ }^{11}$ which found significant improvement in voice quality among teachers with symptoms of vocal fatigue after being exposed to a two days direct treatment in the form of vocal loading exercise. This is also consistent with the findings of Roymet al., ${ }^{12}$ who studied the effectiveness of three treatments for teachers with voice disorders in a randomized clinical trial. Teachers who were experiencing voice problems at the time of the study and/or regularly had experienced voice problems in the past were randomly assigned to three treatment groups for six weeks of therapy: voice amplification using the ChatterVox portable amplifier, resonant voice therapy and respiratory muscle training. Those participants who received the ChatterVox portable amplifier or resonant voice therapy reported significant lowering of their degree of voice handicap and voice disorders severity. The results further revealed that hypothesis two was not rejected because there was no interactive effect of gender and treatment on self perceptive symptoms of vocal fatigue among teachers. Hypothesis three was not rejected because the findings revealed that there was no interactive effect of years of teaching experience and treatment on self perceptive symptoms of vocal fatigue among teachers. ${ }^{13-19}$

\section{Conclusion}

This study confirmed that vocal hygiene training and resonant voice therapy are effective in reducing self perceptive symptoms of vocal fatigue among teachers. Resonant voice therapy was more effective than vocal hygiene training and this confirmed that direct method of treatment is more effective than an indirect mode of treatment of vocal fatigue among teachers. It is recommended that speech and language pathologist should employ the use of direct and indirect interventions in the treatment of vocal fatigue among teachers and other professional voice users. Basics of vocal health and vocal hygiene training modules should be introduced into teacher training programmes in tertiary institutions. Workshops and seminars on vocal hygiene should be organised periodically to equip teachers with skills to help them take care of their voice. Amplification systems should be provided in schools to help reduce the effect of vocal loading among teachers. Teachers and other professional voice users with symptoms of vocal fatigue or other voice disorders should see an ENT (ear, nose and throat) doctor, and a speech and language pathologist with expertise in voice habilitation and rehabilitation for diagnosis and treatment.

\section{Acknowledgments}

None.

\section{Conflicts of interest}

Author declares there are no conflicts of interest.

\section{Funding}

None.

\section{References}

1. Welham NV, Maclagan MA. Vocal fatigue: current knowledge and future directions. $J$ Voice. 2003;17(1):21-30.

2. Hunter EJ. General Statistics of the NCVS Self-Administered Vocal Rating (SAVRa). The National Center for Voice and Speech Online Technical Memo. 2008.

3. Nanjudeswaran C, Vendolini K, Jacobson B. Development and Validation of Vocal Fatigue Index. 2008.

4. Laukkanen $\mathrm{AM}$, Järvinen $\mathrm{K}$, Artkoski $\mathrm{M}$, et al. Changes in voice and subjective sensations during a 45 -min vocal loading test in female subjects with vocal training. Folia Phoniatr Logop. 2004;56(6):335-346.

5. Chan RW. Does the voice improve with vocal hygiene education? A study of some instrumental voice measures in a group of kindergarten teachers. J Voice. 1994;8(3):279-291.

6. Comins R. Voice Forum: Vocal tuition for professional voice users: a tutor's account. Voice. 1995;4:32-43.

7. Rossiter D, Howard DM, Comins R. Objective measurement of voice source and acoustic output change with a short period of vocal tuition. Voice. 1995;4:16-31.

8. Stemple JC, Glaze LE, Gerdman BK. Clinical voice pathology: Theory and management (2nd edn). CA: Singular Publishing Group, San Diego, USA. 1995. 
9. Roy N, Merrill RM, Thibeault S, et al. Voice disorders in teachers and general population: Effects on work performance, attendance, and future career choices. J Speech Lang Hear Res. 2004;47(3):542-551.

10. Stemple JC, Stanley J, Lee L. Objective measures of voice production in normal subjects following prolonged voice use. $J$ Voice. 1995;9(2):127-133.

11. Titze IR, Hunter EJ, Svec JG. Voicing and silence periods in daily and weekly vocalizations of teachers. J Acoust Soc Am. 2007;121(1):469-478.

12. Roy N, Merrill RM, Thibeault S, et al. Prevalence of voice disorders in teachers and general population. $J$ Speech Lang Hear Res. 2004;47(2):281-293.

13. Chang A, Karnell MP. Perceived phonatory effort and phonation threshold pressure across a prolonged voice loading task: a study of vocal fatigue. $J$ Voice. 2004;18(4):454-466.

14. Hunter EJ, Titze IR. Refinements in modeling the passive properties of laryngeal soft tissue. J Appl Physiol. 2007;103(1):206-219.
15. Daniel J McCabe, Ingo R Titze. Chant therapy for treating vocal fatigue among public school teachers: a preliminary study. American Journal of Speech-Language Pathology. 2002;11(4):356-369.

16. Eeva Pekkarinen, Lea Himberg, Jaana Pentti. Prevalence of vocal symptoms among teachers compared with nurses: A questionnaire study. Scand J Log Phon. 1992;17(2):113-117.

17. Roy N, Gray SD, Simon M, et al. An evaluation of the effects of two treatment approaches for teachers with voice disorders: A prospective randomized clinical trials. J Speech Lang Hear Res. 2001;44(23):286-296.

18. Smith E, Gray SD, Dove H, et al. Frequency and effects of teachers' voice problems. $J$ Voice. 1997;11(1):81-87.

19. Smith E, Kirchner H, Taylor M, et al. Voice problems among teachers: Differences by gender and teaching characteristics. $J$ Voice. 1998;12(3):328-334. 